\title{
Balance hídrico superficial mediante el método del índice climático del Valle del Mantaro, 2010 al 2013
}

\section{Superficial water balance by the climate index method from Mantaro Valley, 2010 to 2013}

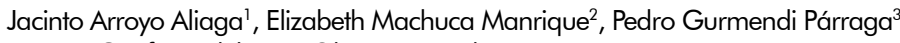
Instituto Geofísico del Perú, Observatorio de Huancayo

\section{RESUMEN}

Objetivos: Determinar el balance hídrico superficial del Valle del Mantaro por cambios en el sistema de evapotranspiración, precipitación y escorrentía durante el período 2010 al 2013. Métodos: Se ha utilizado el método de evaluación de índices que permite analizar el déficit estacional de agua, deducidos del planteamiento de Thornthwaite. Se ha calculado el índice hídrico, analizando el índice de humedad y el índice de aridez a partir de los valores de temperatura media mensual. El índice anual de calor fue calculado teniendo en cuenta el valor de " $a$ " conocido como índice de aridez cuyo valor constante se obtiene para el Valle del Mantaro. Resultados: Se determinó el valor del índice hídrico superficial anual en $\mathrm{Im}=55,72$, el índice de humedad $\mathrm{lh}=42,7$, el índice de aridez la $=-21,71$ y la escorrentía anual $Q a=412 \mathrm{~mm}$. Conclusiones: El balance hídrico superficial del Valle del Mantaro, muestra en función del índice de aridez un déficit estacional de agua para los meses de junio, julio, agosto y setiembre que corresponde al invierno y en función del índice de humedad muestra un exceso para los meses de diciembre, enero, febrero y marzo que corresponde al verano; estos valores se contrastan con el coeficiente del déficit de agua para los meses de mayo y se prolonga hasta el mes de setiembre, abarcando desde la temporada de finales del otoño hasta los finales del invierno.

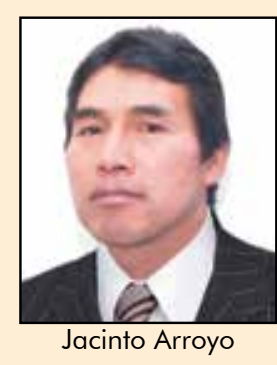

jarroyox@gmail.com

Palabras clave: Balance hídrico superficial, índice climático, Valle del Mantaro.

1 Investigador del Observatorio de Huancayo, Instituto Geofísico del Perú.

2 Investigadora de la Universidad Alas Peruanas.

3 Docente de la Universidad Continental. 


\section{ABSTRACT}

Objectives: To determine the superficial water balance from Mantaro Valley because of evapotranspiration system changes, precipitation and runoff during the periods from 2010 to 2013. Methods: We used the indices evaluation method, which allows to analyze the seasonal water deficit, deducted from the Thornthwaite approach. We calculated the water index, analyzing the humidity and aridity indices from the monthly mean temperature values. The warm annual index has been calculated taking into account the " $a$ " value, known as aridity index which constant value is obtained for Mantaro Valley. Results: The annual superficial water index value was determined in $\mathrm{Im}=55,72$, the humidity index in $\mathrm{lh}=42,7$, the aridity index in la $=$ $-21,71$ and the annual runoff in $Q a=412$ $\mathrm{mm}$. Conclusions: In function of the aridity index, the Mantaro Valley superficial hydric balance shows a water seasonal deficit for the months of june, july, august and september which corresponds to winter; and in function of the humidity index shows an excess for the months of december, january, february and march which corresponds to summer; these values are compared with the water deficit coefficient for may and lasts until september, covering from the late fall season to the end of winter.

Keywords: Superficial hydric balance, climate index, Mantaro Valley.

\section{INTRODUCCIÓN}

La escasez de agua es una amenaza significativa y creciente para el ambiente, la salud humana, la seguridad energética y el abastecimiento mundial de alimentos (1). El agua dulce que sustentan la vida, están siendo sujetos a múltiples presiones, entre las que se incluye la necesidad de agua de calidad adecuada y en la cantidad necesaria en el momento apropiado. Pero, la escasez del agua constituye un factor significativo para la seguridad hídrica humana; ya que, una quinta parte de la población del mundo habita en áreas con escasez de agua (2). Se estima que el agua requerida para mantener bienes y servicios asciende al $75 \%$ del uso total, mientras que el uso directo por el hombre representa solo el $25 \%$ (3). Estas cifras incluyen tanto las aguas subterráneas y superficiales así como el agua almacenada en el suelo. Un estudio de 424 de las principales cuencas fluviales que albergan a una población de 3900 millones de personas concluyen que enfrentan una escasez severa de este recurso durante por lo menos un mes al año (4). También se reportan que en regiones áridas del norte de África y del Medio Oriente el agua renovable que se extrae en estas regiones es mayor al $50-75 \%$, lo que deja un flujo ambiental pequeño (5).

La extracción de agua a nivel mundial se ha triplicado durante los últimos 50 años (6) a fin de satisfacer las demandas de una población cada vez mayor con niveles crecientes de bienestar y consumo del líquido. Aunque el abastecimiento de agua durante este periodo ha permanecido relativamente constante, actualmente la demanda rebasa el suministro sostenible en muchas áreas, lo cual conlleva serias implicaciones a largo plazo (7). Se estima que el límite planetario para el consumo humano de agua dulce cuando los acuíferos y las aguas superficiales no pueden reutilizarse en la misma cuenca es de 4000 km3 por año, con un consumo de agua dulce estimado de aproximadamente 2600 km3 por año. Según Bates (8) es probable que las demandas de agua proyectadas alcancen los límites planetarios en las siguientes décadas.

En el Perú las extracciones de agua para usos agrícolas, industriales y domésticos han aumentado gradualmente. La agricultura es el mayor usuario del recurso hídrico a nivel nacional (9), y las extracciones para este fin son insostenibles en muchas áreas debido a un balance hídrico para riego desequilibrado a largo plazo (10), como lo demuestra la explotación de acuíferos y la dependencia de grandes proyectos 
de desviación de agua que se vienen realizando.

La situación de los valles interandinos también es preocupante, estos se ven sometidos a fuertes presiones hídricas en tres actividades principales, el consumo humano, la agricultura y la industria que aumenta a medida que lo hace la demanda de la población. Pero, żen qué medida se conoce el balance hídrico en el Valle del Mantaro que permita planificar un uso equilibrado? y żde qué manera el balance hídrico superficial del Valle del Mantaro se altera por cambios en los sistemas del flujo hidrológico?

El objetivo de la investigación fue determinar el balance hídrico superficial del Valle del Mantaro por cambios en el sistema de evapotranspiración, precipitación y escorrentía en los periodos del año 2010 al 2013.

Este propósito debido a que el agua en la naturaleza se presenta bajo diversas formas y aspectos; sin embargo, hay un orden, una secuencia natural del pasaje de una forma a otra que obedecen al ciclo natural del agua (11). Todos estos cambios y diferentes formas que afecta el agua en la naturaleza constituyen lo que se conoce como el ciclo hidrológico.

Los sistemas de evapotranspiración, precipitación y escorrentía presentan cambios estacionales que se deben a la estacionalidad hídrica anual, la cual repercute en un déficit de agua en los meses que corresponden al invierno. Estos mismos sistemas presentan un exceso hídrico en los meses de verano y que tienden a un estrés hídrico en los meses de primavera. Sin embargo, estas distribuciones se han ido modificando durante el periodo 2010 - 2013.

\section{MATERIAL Y MÉTODOS}

Se ha recurrido al método deductivo- hipotético del tipo de investigación aplicada y nivel explicativo, haciendo uso de las estrategias de diseño no experimental. La obtención de información fue por medios directos y complementados con medios indirectos de documentación obtenida de centros y agencias de investigación. En el tratamiento de información se ha recurrido al método del análisis de la información de precipitación, escorrentía superficial y de evapotranspiración potencial.

Para el análisis de la precipitación utilizamos el mapa de isoyetas de la región Junín y 3 registros de lluvias de estaciones localizadas en el Valle. Luego verificamos la calidad, relleno y extensión de registros de lluvia mensual y anual, basándonos en las técnicas de la evaluación espacial. Para la verificación de la lluvia anual usamos el método de curvas de doble acumulación (CDA) y para el cálculo del patrón de precipitación anual (PPA) tomamos el promedio de los datos anuales de precipitación de las tres estaciones y establecimos una correlación con estaciones semejantes.

Las ecuaciones utilizadas para la extensión y relleno de la información fueron (12).

$P_{x}=\frac{\bar{P}_{x}}{3}\left(\frac{P_{a}}{P_{a}}+\frac{P_{b}}{P_{b}}+\frac{P_{c}}{P_{c}}\right)$

Donde:

$\mathrm{P}_{\mathrm{a}^{\prime}} \mathrm{P}_{\mathrm{b}} \mathrm{y}_{\mathrm{c}^{\prime}}=$ promedios de las precipitaciones anvales registradas en las estaciones $A, B$, $\mathrm{C}$ de un periodo común.

Para la determinación de la escorrentía directa se ha utilizado la siguiente relación (13):

$$
\frac{P-Q}{S}=\frac{Q}{P}
$$

Donde:

$Q=$ Escorrentía directa en lámina de agua $\mathrm{P}=$ Precipitación en lámina de agua 
$S=$ Diferencia potencial máxima entre $P y, Q$, a la hora que comienza las precipitaciones. $Q$ se aproxima a $P$, mientras $P$ aumenta. También los valores ( $P-Q$ ) se aproximan a una constante mientras $\mathrm{P}$ continúa aumentando. Además ( $P-Q$ ) real está limitado por el agua almacenada en el suelo o por la intensidad de la infiltración al aumentar P. El potencial máximo $(P-Q) \circ S$, por lo tanto depende del agua almacenada en el suelo y de las intensidades de infiltración de una cuenca (14).

$$
Q=\frac{P^{2}}{P+S}
$$

Haciendo uso de la precipitación que ocurre antes de que comience el escurrimiento $\left(I_{a}\right)$, $Q$, queda.

$$
Q=\frac{\left(P-I_{a}\right)^{2}}{P-I_{a}+S}
$$

Para el cálculo de la evapotranspiración como método específico se ha recurrido al modelo del índice climático, planteado por Thornthwaite (15) y modificado por Penman - Monteith (16). Este método puede considerarse como el estándar de todos los métodos combinados para estimar la evapotranspiración (ET). La mayoría de los métodos combinados presentan ligeras diferencias dependiendo de la localización de los instrumentos meteorológicos. Por esta razón, el método de Penman-Monteith utiliza términos como la resistencia aerodinámica de los vegetales para relacionar la altura de los instrumentos meteorológicos con la altura de dichas especies, en tal sentido la resistencia estomática a la transpiración mínima dependerá de su altura. Siendo la ecuación la siguiente:

$\mathrm{ET}_{\mathrm{O}}=\left[\frac{\Delta}{\Delta+\gamma^{*}}(\mathrm{Rn}-\mathrm{G}) \frac{10}{\mathrm{~L}}+\frac{\gamma}{\Delta+\gamma^{*}} \frac{90}{\mathrm{~T}+275} \mathrm{U}_{2}\left(\mathrm{e}_{\mathrm{s}}-\mathrm{e}_{\mathrm{a}}\right)\right]$

Donde:

$\mathrm{ET}_{\mathrm{o}}=$ evapotranspiración ( $\mathrm{mm} /$ día)

$\gamma^{*}=$ constante psicométrica modificada utilizada en el método de PenmanMonteith (mbar/C) $\mathrm{e}_{\mathrm{s}}-\mathrm{e}_{\mathrm{a}}=$ déficit de presión de vapor $(\mathrm{mb})$

$\mathrm{e}_{\mathrm{s}}=$ presión de vapor a saturación a la temperatura promedio del aire $(\mathrm{mb})$

$\mathrm{e}_{\mathrm{a}}=$ presión de vapor tomada a la temperatura a punto de rocío (mb)

$\mathrm{L}=$ calor latente de vaporización (cal/gr)

$\Delta=$ pendiente de la curva de presión de la saturación de vapor a una temperatura específica $\left(\mathrm{mbar} /{ }^{\circ} \mathrm{C}\right)$

$\gamma=$ constante psicométrica

$\mathrm{R}_{\mathrm{n}}=$ energía de radiación neta $\left(\mathrm{cal} /\left(\mathrm{cm}^{2}\right.\right.$ día)

$\mathrm{T}=$ temperatura promedio $\left({ }^{\circ} \mathrm{C}\right)$

$\mathrm{G}=$ flujo termal del suelo $\left(\mathrm{cal} / \mathrm{cm}^{2}\right)$

Finalmente la diferencia entre la precipitación del lugar de análisis y la evapotranspiración potencial mensual estacional o anual, adquiere valores positivos o negativos. $\mathrm{Si}$ es positiva hay superávit o exceso (s) de agua y si es negativa hay déficit o falta (d). Si la diferencia obtenía corresponde al valor de cero entonces resulta que la precipitación resulta exactamente igual a la necesidad (n) de agua, es decir, a la evapotranspiración potencial (e). Seguidamente se deben igualar los valores de $(e)=(s)$, e inmediatamente se deducen a (d) y (n) que muestran los índices siguientes:

Índice de humedad $I_{h}=100-\frac{\Sigma^{s}}{\text { ETP anual }}$

Índice de aridez $\quad \mathrm{I}_{\mathrm{a}}=100-\frac{\Sigma^{\mathrm{d}}}{\text { ETP anual }}$

Del cálculo de estos dos índices para el año, se obtiene:

Índice hídrico anual $I_{m}=I_{h}-0,61_{a}$

\section{RESULTADOS}

\section{Análisis del índice hídrico anual}

El análisis del balance hídrico anual, como se presenta en la tabla $N^{\circ} 1$, muestra 
Tabla $N^{\circ}$ 1: Análisis del índice hídrico anual

\begin{tabular}{|c|c|c|c|c|c|c|c|c|c|c|c|c|}
\hline Meses & $E$ & $F$ & $M$ & A & $M$ & $\mathrm{~J}$ & $J$ & A & $S$ & O & $N$ & D \\
\hline Temperatura & 11,80 & 11,70 & 11,50 & 11,20 & 10,30 & 9,40 & 9,30 & 10,40 & 11,50 & 12,10 & 12,30 & 12,00 \\
\hline $\mathrm{Pp}$ & 120,00 & 122,00 & 111,00 & 55,00 & 21,00 & 8,00 & 7,00 & 16,00 & 44,00 & 67,00 & 69,00 & 92,00 \\
\hline días & 31,00 & 28,00 & 31,00 & 30,00 & 31,00 & 30,00 & 31,00 & 31,00 & 30,00 & 31,00 & 30,00 & 31,00 \\
\hline$x P p$ & 3,87 & 4,35 & 3,57 & 1,83 & 0,67 & 0,27 & 0,24 & 0,51 & 1,48 & 2,15 & 2,30 & 2,97 \\
\hline$i=$ & 3,68 & 3,60 & 3,52 & 3,40 & 3,00 & 2,59 & 2,55 & 3,02 & 3,51 & 3,80 & 3,91 & 3,77 \\
\hline $\mathrm{ETP}=$ & 54,00 & 53,10 & 52,20 & 50,80 & 46,30 & 41,50 & 41,10 & 46,60 & 52,10 & 55,30 & 56,50 & 55,00 \\
\hline $\mathrm{DIF}=$ & 66,00 & 68,70 & 58,30 & 4,00 & $-25,40$ & $-33,50$ & $-33,70$ & $-30,80$ & $-7,80$ & 11,40 & 12,50 & 37,10 \\
\hline Coeficientes & $\mathrm{s}$ & s & s & $\mathrm{s}$ & $d$ & $d$ & $d$ & $d$ & $d$ & s & s & s \\
\hline Déficit estacional & 1,223 & 1,294 & 1,118 & 0,078 & $-0,548$ & $-0,806$ & $-0,821$ & $-0,662$ & $-0,149$ & 0,206 & 0,222 & 0,675 \\
\hline
\end{tabular}

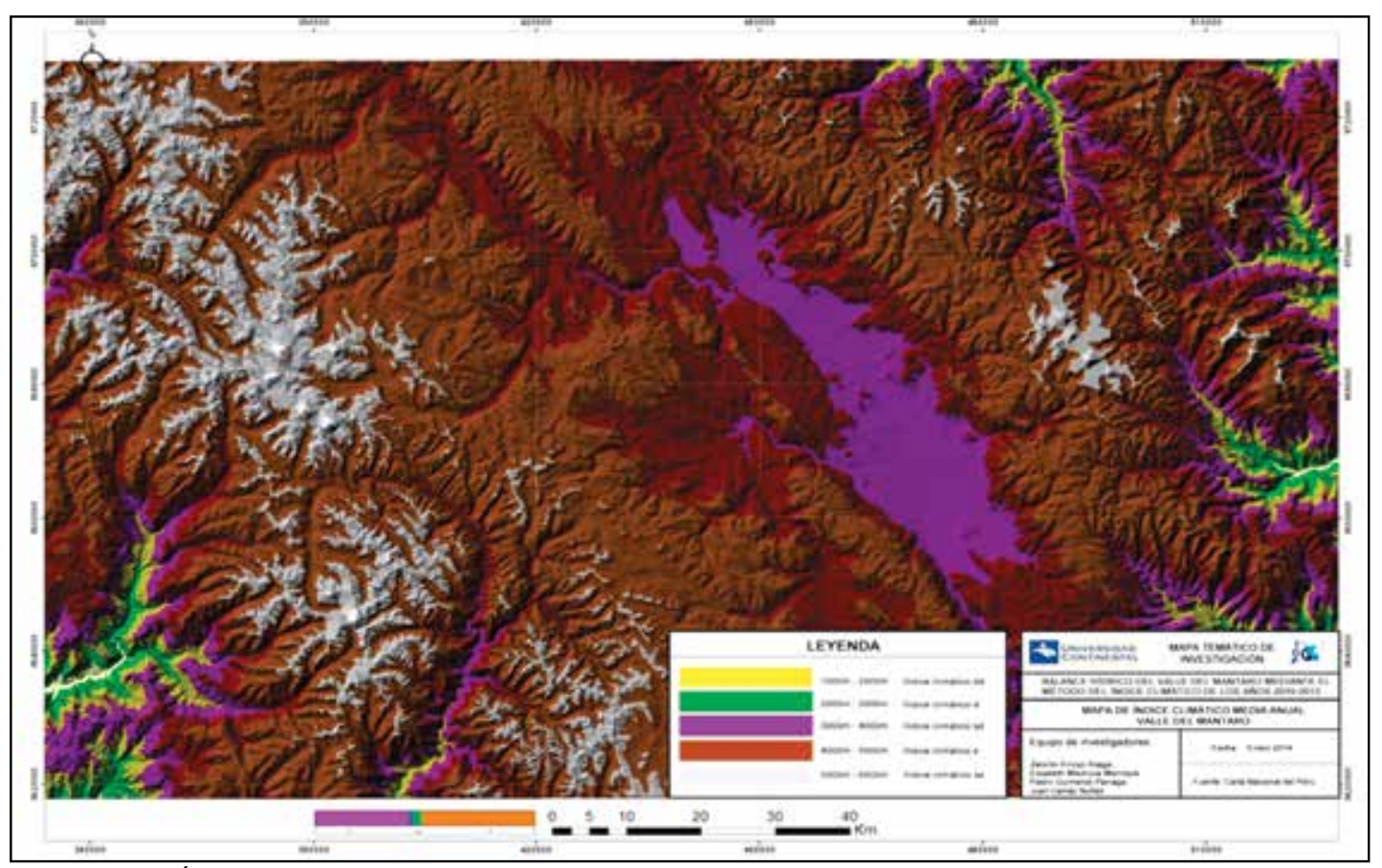

Figura $\mathrm{N}^{\circ} 1$ : Índice hídrico anual para el Valle del Mantaro años 2010 - 2013

los coeficientes de aridez (s) y humedad (d) que indican las variaciones hídricas estacionales en función a la precipitación (Pp), índice de calor (i) y evapotranspiración (ETP). Los valores negativos obtenidos de la diferencia del índice precipitación (xPp) y los valores de ETP, indican deficiencias hídricas denominados stress hídrico por no tener recarga.

En la figura $\mathrm{N}^{\circ} 1$ se muestra el mapa del índice hídrico anual en una distribución espacial con deficiencias de aridez (s) y semiaridez (sd) en las zonas altas del Valle del Mantaro y con exceso de humedad (d) en las zonas bajas.
En la figura $N^{\circ} 2$ se presenta la distribución promedio mensual de la precipitación en el Valle del Mantaro. Las precipitaciones más altas se concentran en los meses de enero, febrero y marzo para luego disminuir en los meses de mayo, junio, julio y agosto. Estas observaciones también se verifican con los modelos del índice climático.

En la figura $\mathrm{N}^{\circ} 3$ se muestra los resultados del análisis de doble masa para las dos estaciones representativas del Valle del Mantaro con el objetivo de dar consistencia a la información obtenida de los isoyetas. Este análisis se basó en el criterio de que los valores acumulados de la precipitación en 


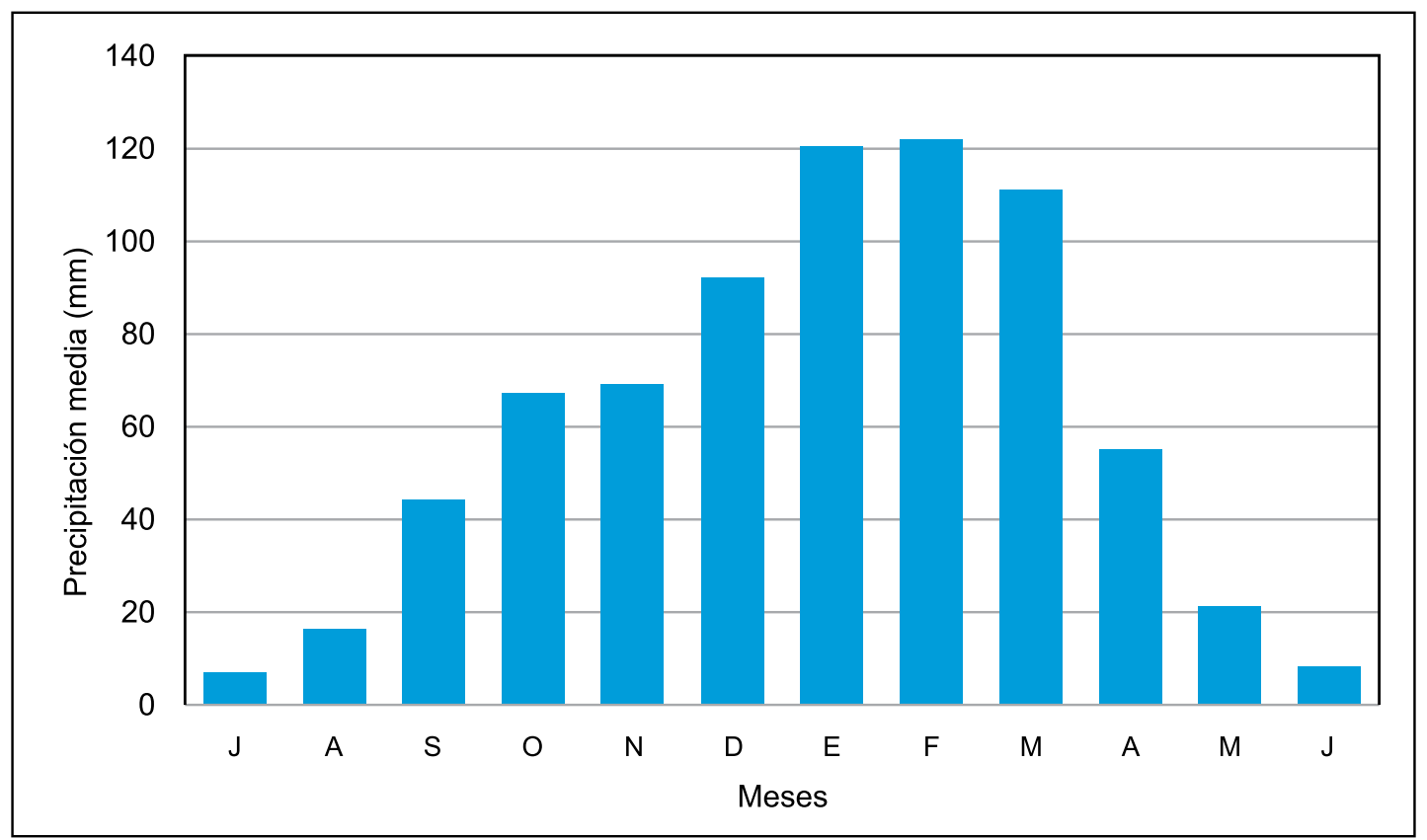

Figura $N^{\circ}$ 2: Distribución promedio de la precipitación mensual para el Valle del Mantaro (2010-2013)

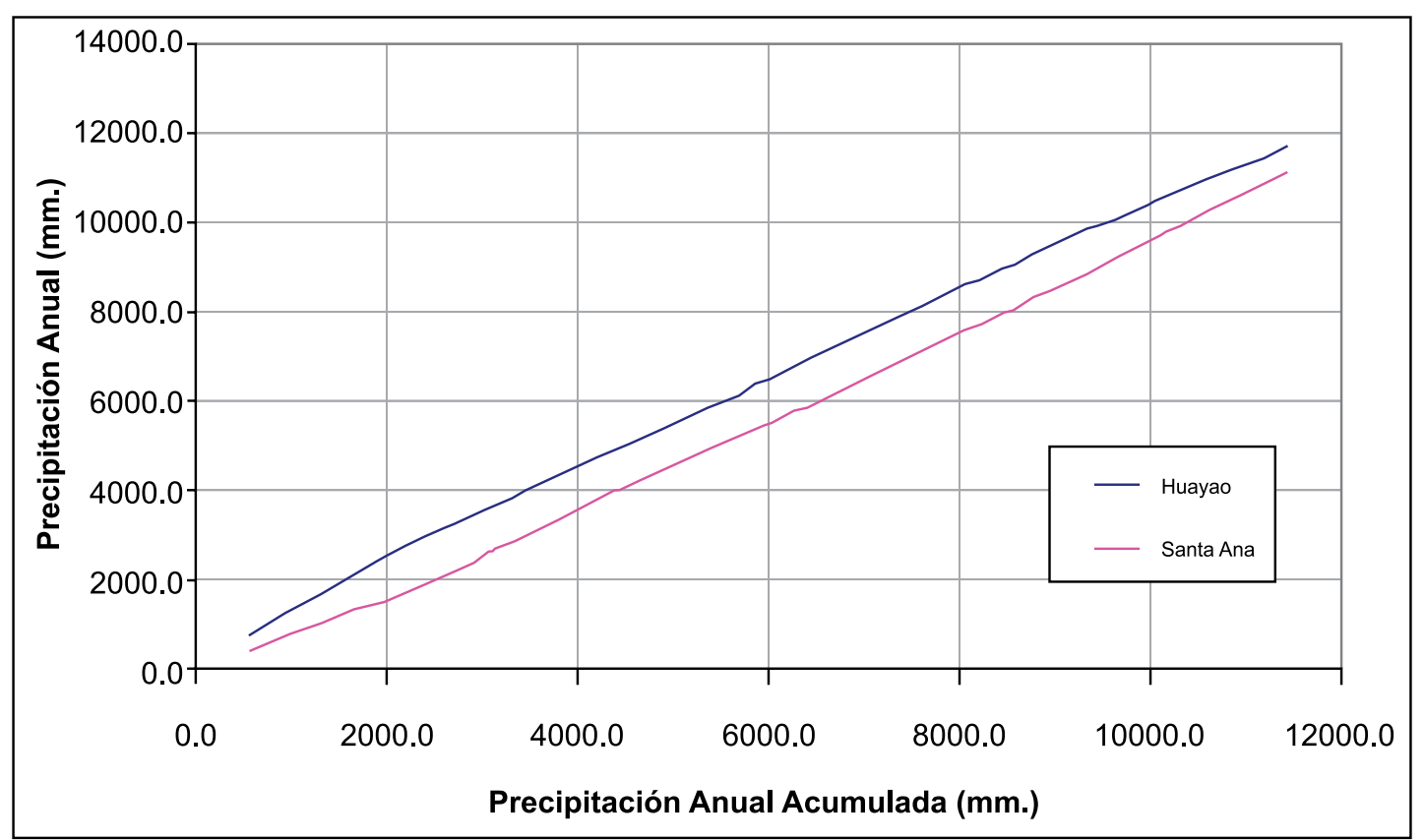

Figura $N^{\circ}$ 3: Diagrama de doble masa para los años 2010-2013

Tabla $N^{\circ} 2$ : Variación estacional de escorrentía

\begin{tabular}{|c|c|c|c|c|c|c|c|c|c|c|c|c|}
\hline Meses & $E$ & $F$ & $M$ & A & $M$ & $\mathrm{~J}$ & $\mathrm{~J}$ & A & $S$ & 0 & $\mathrm{~N}$ & D \\
\hline $\mathrm{Pp}$ & 120,00 & 122,00 & 111,00 & 55,00 & 21,00 & 8,00 & 7,00 & 16,00 & 44,00 & 67,00 & 69,00 & 92,00 \\
\hline$Q$ & 53,00 & 67,00 & 96,00 & 50,00 & 19,00 & 4,00 & 0,00 & 0,00 & 0,00 & 28,00 & 2,00 & 92,00 \\
\hline $\mathrm{ETP}=$ & 54,00 & 53,10 & 52,20 & 50,80 & 46,30 & 41,50 & 41,10 & 46,60 & 52,10 & 55,30 & 56,50 & 55,00 \\
\hline Déficit & 66,00 & 68,70 & 58,30 & 4,00 & $-25,40$ & $-33,50$ & $-33,70$ & $-30,80$ & $-7,80$ & 11,40 & 12,50 & 37,10 \\
\hline
\end{tabular}




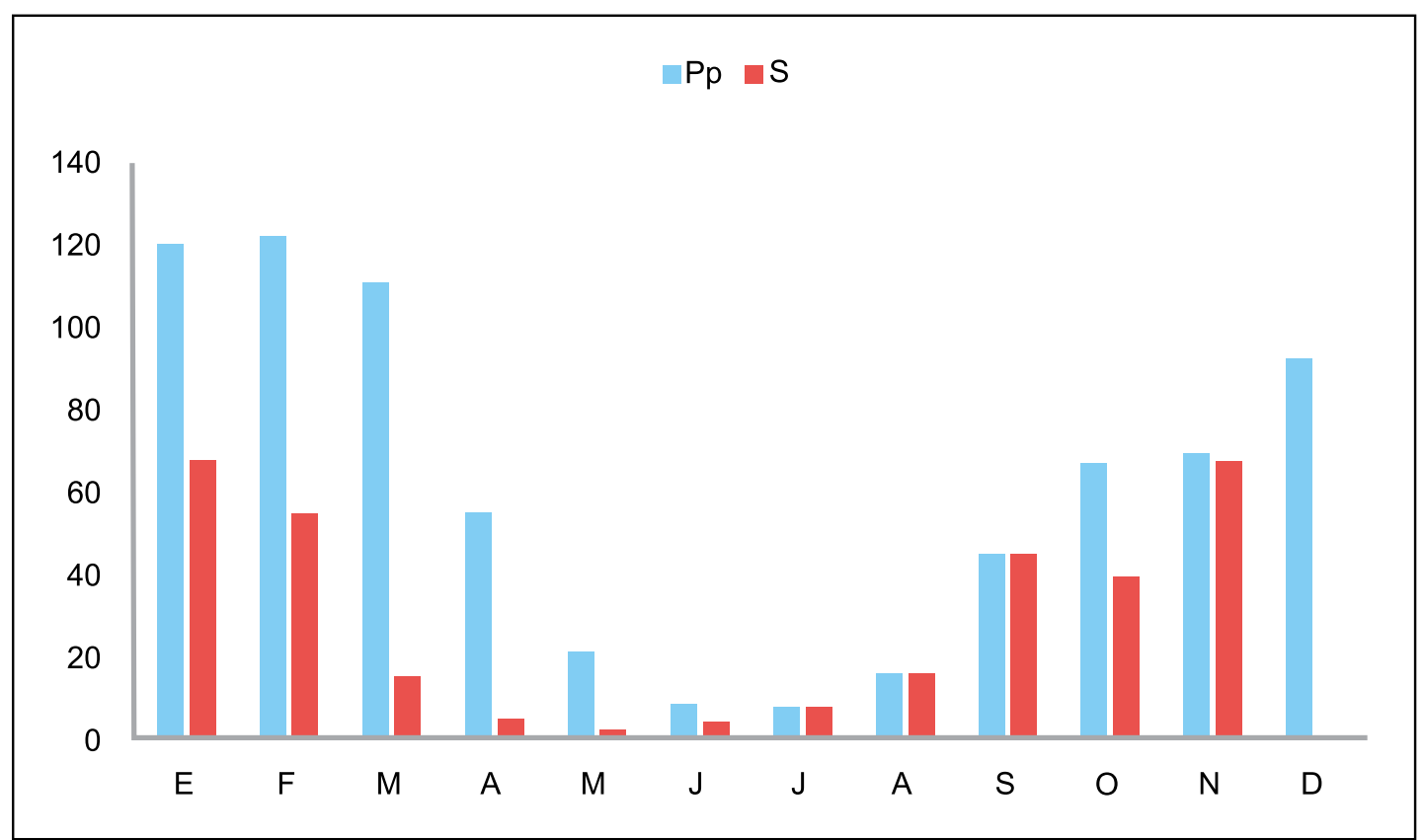

Figura $N^{\circ}$ 4: Diferencias mensuales entre la precipitación (Pp) y la escorrentía (S)

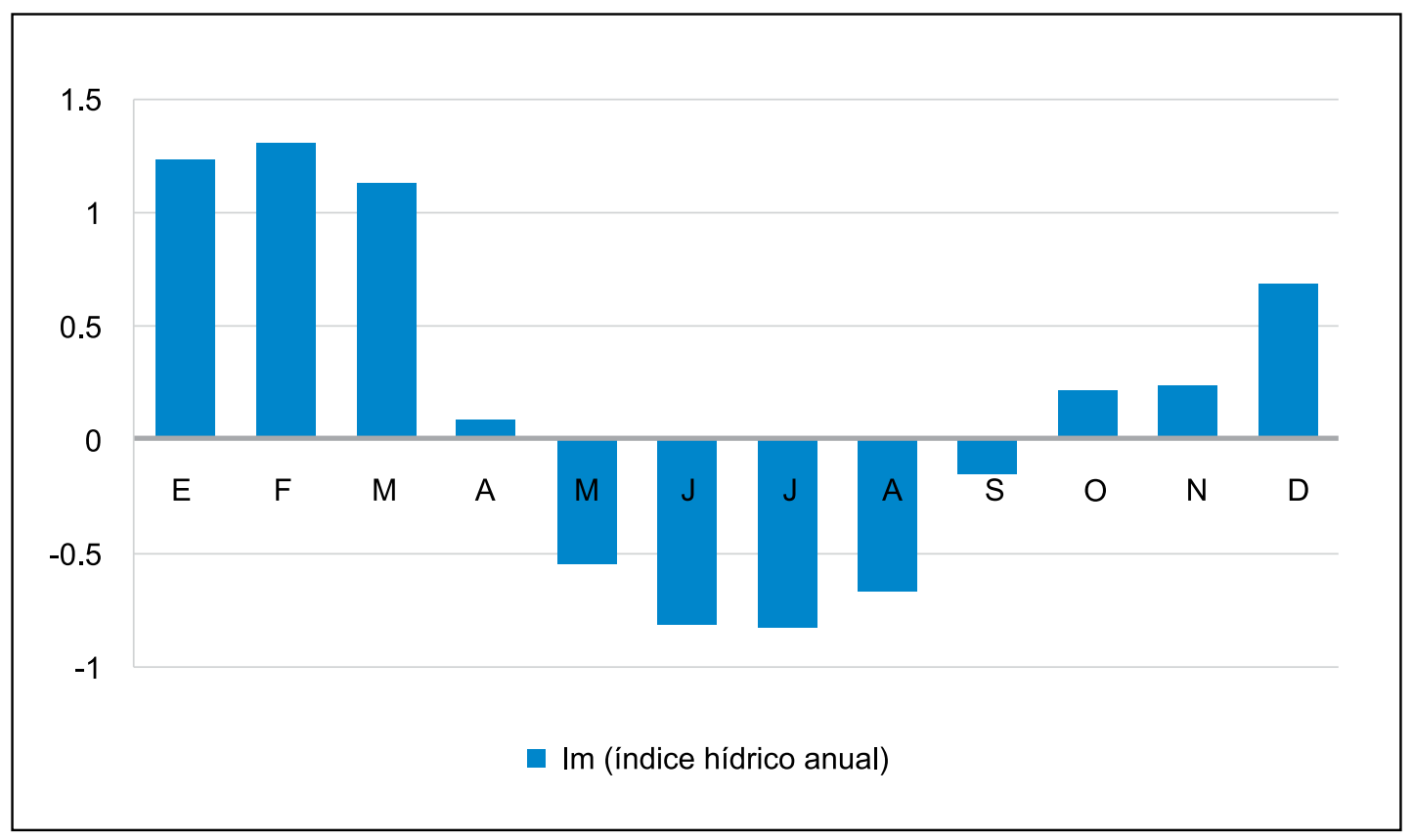

Figura $\mathrm{N}^{\circ}$ 5: Déficit estacional de agua a partir de las precipitaciones y del ETP (Método de Thornthwaite)

cada estación se comparan con la estación modelo. Las líneas deben ser rectas de pendiente constante que sirve para realizar inferencia de su consistencia.

\section{Análisis de escorrentía}

La tabla $N^{\circ} 2$ muestra los resultados de la escorrentía ( $Q$ ) estacional que se presentan durante los años de análisis. La escorrentía anual fue estimada en $Q^{\dagger}=412 \mathrm{~mm}$ y el valor de la diferencias (S) entre la precipitación y la escorrentía equivale a $\mathrm{S}=$ $(P-Q)=320 \mathrm{~mm}$.

En la figura $\mathrm{N}^{\circ} 4$ se observa las diferencias significativas de la precipitación y la escorrentía. 


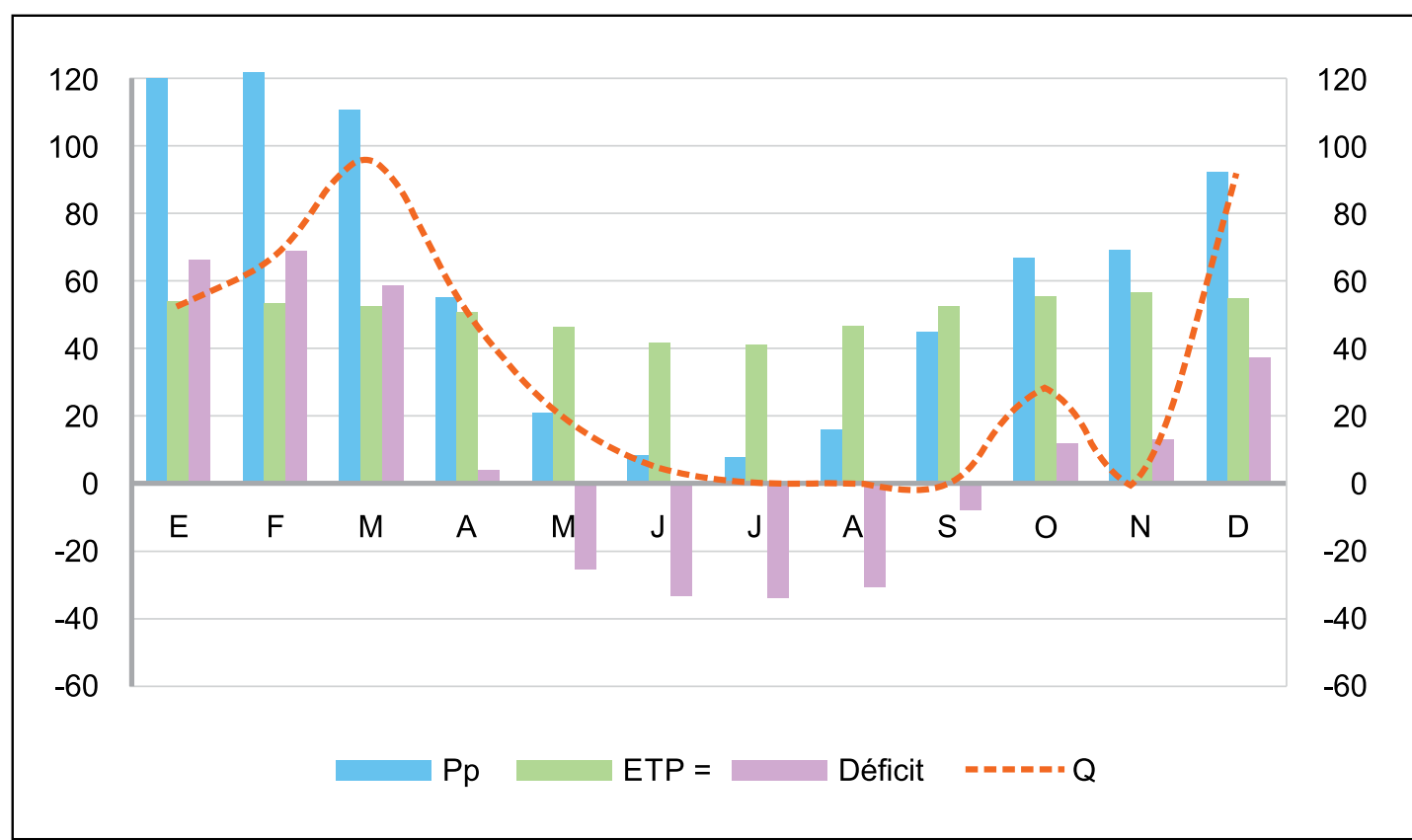

Figura $\mathrm{N}^{\circ}$ 6: Comportamiento estacional de la precipitación, ETP, escorrentía y déficit estimado en los años de análisis

\section{Análisis del índice hídrico anual}

El índice hídrico anual, como se muestra en la figura $\mathrm{N}^{\circ} 5$ muestra el déficit estacionario para los meses comprendidos de mayo, junio, julio, agosto y parte de setiembre. Los meses con excesos de humedad corresponden a los meses de enero, febrero y mayo. Además se muestra los procesos de inversión que suceden al inicio del otoño, cuando empiezan a disminuir las precipitaciones, y a finales de la primavera e inicios del verano, cuando se incrementan las precipitaciones y disminuye la evapotranspiración.

\section{Comportamiento de variables que regulan el índice hídrico anual}

La figura $N^{\circ} 6$ muestra el comportamiento de las principales variables de evapotranspiración que tienen un comportamiento inversamente proporcional a las precipitaciones de la temporada. La escorrentía que es directamente proporcional a la precipitación y el déficit de agua para los meses del invierno.

\section{DISCUSIÓN}

El balance hídrico superficial del Valle del Mantaro, muestra una tendencia estacional a un déficit de agua en los sistemas (tabla $N^{\circ} 1$ ), el déficit de agua es mayor en los meses de mayo, junio, julio, agosto y setiembre. Pero en los meses de diciembre, enero, febrero y marzo existe un exceso de humedad, dónde los sistemas colapsan con un incremento sobre su equilibrio estacional. También el coeficiente hídrico es negativo a mayores altitudes y el coeficiente se vuelve positivo a menor altitud (figura $\mathrm{N}^{\circ}$ 1), que contribuye de esta manera que el sistema llegue a un equilibrio dinámico de acuerdo a la conservación de masas.

Los procesos de análisis de precipitación implicaron las técnicas de mediciones de almacenamiento y flujos de agua con los sistemas de estudio. Sin embargo, algunas mediciones se eliminaron en función del volumen y periodo de tiempo utilizados para el cálculo del balance superficial (figura $N^{\circ} 2$ ). La ecuación de la precipitación representó significativamente las áreas de estudio y se puede aplicar para cualquier zona o masa de agua (figura $\mathrm{N}^{\circ} 3$ ) porque 
indica valores relativos de entrada y salida del flujo y la variación del volumen de agua almacenada en las zonas o masas de agua del sistema.

La escorrentía superficial anual (Q) es superior a la diferencia (S) que existe entre la precipitación y escorrentía estacional. Esto indica que gran parte de las entradas de flujos de agua por precipitación, se pierde por escurrimiento aumentando el caudal de los ríos (tabla $\mathrm{N}^{\circ} 2$ ). La retención hídrica neta equivale a $320 \mathrm{~mm}$ anual, esto es un indicador de la baja capacidad de retención hídrica que tienen los suelos del Valle del Mantaro. El flujo neto de pérdida de agua equivale a $412 \mathrm{~mm}$, superior a la capacidad de retención.

La distribución espacial de la precipitación corresponde a los meses del verano lluvioso (figura $\mathrm{N}^{\circ} 2$ ), condición básica para que en estos meses, no exista déficit hídrico. Sin embargo, los déficit hídricos significativos, se presentan en los meses que corresponden al invierno, en donde solo existe pérdida por evapotranspiración (figura $\mathrm{N}^{\circ} 4$ ). Los factores de la variabilidad de la precipitación generan fuertes desbalances hídricos (figura $\mathrm{N}^{\circ}$ 5).

El comportamiento estacional de la precipitación, la escorrentía y la evapotranpiración condicionan el déficit hídrico del Valle del Mantaro alrededor de los $40 \mathrm{~mm}$. Esto concuerda con la estación seca o de estiaje que se presenta. El déficit de agua representa el $40 \%$ de la capacidad de retención de los suelos (figura $N^{\circ} 6$ ).

\section{Agradecimientos}

A Rosalind y Edsel Arroyo por su apoyo en la recopilación de información, a Gaudencio Quispealaya por su apoyo en la sistematización de la información.

En el ámbito institucional, al Instituto Geofísico del Perú por el apoyo científico en la investigación, y a la Universidad Continental por el apoyo en la publicación.

\section{REFERENCIAS} BIBLIOGRÁFICAS

1. 2030 Water Resources Group. Charting our Water Future: Economic Frameworks to Inform Decision-Making. 2009. Disponible en: http://www.mckinsey. com/App Media/Reports/Water/ Charting_Our_Water_Future_Full_ Report_001.pdf

2. Brauch HG, Oswald Spring U, Grin J, Mesjasz C, Kameri-Mbote P, Behera NC, et al, eds. Facing Global Environmental Change: Environmental, Human, Energy, Food, Health and Water Security Concepts. Springer-Verlag, Berlin; Heidelberg, New York; 2009.

3. Alcamo J, Van Vuuren DP, Cramer W. Change in ecosystem services and their drivers across the scenarios. In Ecosystems and Human Well-being: Scenarios. Volume 2 (Carpenter SR, Pingali P, Bennett EM, Zurek MB, eds.). Island Press, Washington, DC; 2005.

4. Ali MH. Fundamentals of Irrigation and On-Farm Water Management, volume 1 , and Practices of Irrigation and OnFarm Water Management, volumen 2. Springer Science+Business Media, New York, NY; 2010.

5. Anderson DM, Reguera B, Pitcher GC y Enevoldsen $\mathrm{HO}$. The IOC international harmful algal bloom program: history and science of impacts. Oceanography 23, 72-85. 2010.

6. Antonov JL, Levitus S, Boyer TP. Thermostatic sea level rise, 19552003. Geophysical Research Letters 32, L12602. 2005.

7. Bakkes JA, Boschet PR, eds. Background Report to the OECD Environmental Outlook to 2030: Overviews, Details, and Methodology of Model-based Analysis. MNP Report 500113001. Netherlands Environmental Assessment Agency (Milieu-en Natuurplanbureau) and Organisation for Economic Cooperation and Development, Paris. 2008.

8. Bates BC, Kundzewicz ZW, Wu S, Palutikof $J P$, eds. Climate Change and Water. Technical paper of Intergovernmental 
Panel on Climate Change. IPCC Secretariat, Geneva. 2008.

9. Bennett V, Dávila-Poblete S, Rico $M N$. Opposing Currents: The Politics of Water and Gender in Latin America. University of Pittsburg Press, Pittsburg, PA. 2005.

10. Bird KJ, Charpentier RR, Gautier DL, Houseknecht DW, Klett TR, Pitman JK, et al. Circum-Arctic Resource Appraisal: Estimates of Undiscovered Oil and Gas North of the Arctic Circle. US Geological Survey Fact Sheet 2008-3049.2008 http://pubs.usgs.gov/fs/2008/3049/

11. Tosi JA. An Ecological Model for the Prediction of Carbon Offsets by Terrestrial Biota -Tropical Science Center. Occasional Paper $N^{\circ}$ 17. San José: Ediciones del Instituto Ecológico de CR. 1997; 34p.

12. Gorman PA, Schneider T. The physical basis for increases in precipitation extremes in simulations of 21 st century climate change. Proceedings of the National Academy of Sciences of the United States of America 106(35), 14773-14777. 2009.

13. Garrick D, Siebentritt MA, Aylward B, Baver DCJ, Purkey A. Water markets and freshwater services: policy reform and implementation in the Columbia and Murray-Darling Basins. Ecological Economics 69, 366-379. 2009.

14. Biswas A.K. Integrated water resources management: a re-assessment. Water International 29(2), 248-256.2010.

15. FAO. FAO-Aquastat: Proportion of Renewable Water Resources Withdrawn (MDG Water Indicator). Food and Agriculture Organization of the United Nations, Rome. 2008. Disponible en: http://www.fao.org/nr/water/aquastat/ globalmaps/index.stm

16. Global Water Partnership. Setting the Stage for Change: Second Informal Survey by the GWP Network Giving the Status of the 2005 WSSD Target on National Integrated Water Resources Management and Water Efficiency Plans. Global Water Partnership, Stockholm. 2006. 